\title{
Understanding the Flowing Atmospheric- Pressure Afterglow (FAPA) Ambient Ionization Source through Optical Means
}

\author{
Jacob T. Shelley, George C.-Y. Chan, Gary M. Hieftje \\ Department of Chemistry, Indiana University, 800 E. Kirkwood Ave., Bloomington, IN 47405, USA
}

\begin{abstract}
The advent of ambient desorption/ionization mass spectrometry (ADI-MS) has led to the development of a large number of atmospheric-pressure ionization sources. The largest group of such sources is based on electrical discharges; yet, the desorption and ionization processes that they employ remain largely uncharacterized. Here, the atmospheric-pressure glow discharge (APGD) and afterglow of a helium flowing atmospheric-pressure afterglow (FAPA) ionization source were examined by optical emission spectroscopy. Spatial emission profiles of species created in the APGD and afterglow were recorded under a variety of operating conditions, including discharge current, electrode polarity, and plasma-gas flow rate. From these studies, it was found that an appreciable amount of atmospheric $\mathrm{H}_{2} \mathrm{O}$ vapor, $\mathrm{N}_{2}$, and $\mathrm{O}_{2}$ diffuses through the hole in the plate electrode into the discharge to become a major source of reagent ions in ADI-MS analyses. Spatially resolved plasma parameters, such as $\mathrm{OH}$ rotational temperature $\left(T_{\text {rot }}\right)$ and electron number density $\left(n_{e}\right)$, were also measured in the APGD. Maximum values for $T_{\text {rot }}$ and $n_{\mathrm{e}}$ were found to be $\sim 1100 \mathrm{~K}$ and $\sim 4 \times 10^{19} \mathrm{~m}^{-3}$, respectively, and were both located at the pin cathode. In the afterglow, rotational temperatures from $\mathrm{OH}$ and $\mathrm{N}_{2}{ }^{+}$yielded drastically different values, with $\mathrm{OH}$ temperatures matching those obtained from infrared thermography measurements. The higher $\mathrm{N}_{2}{ }^{+}$temperature is believed to be caused by charge-transfer ionization of $\mathrm{N}_{2}$ by $\mathrm{He}_{2}{ }^{+}$. These findings are discussed in the context of previously reported ADIMS analyses with the FAPA source.
\end{abstract}

Key words: Ambient desorption/ionization mass spectrometry, Flowing atmospheric-pressure afterglow, Atmospheric-pressure glow discharge, Optical plasma diagnostics

\section{Introduction}

$\mathrm{R}$ ecently, a large number of atmospheric-pressure ionization sources has been introduced that enable direct analysis of samples for detection by mass spectrometry. This emerging technology has resulted in a new field termed

Electronic supplementary material The online version of this article (doi:10.1007/s13361-011-0292-8) contains supplementary material, which is available to authorized users.

Correspondence to: Gary M. Hieftje; e-mail: hieftje@indiana.edu ambient desorption/ionization mass spectrometry (ADI-MS). Since the introduction of the field in $2004[1,2]$, over 30 ambient desorption/ionization sources have been described in the literature [3, 4]. While the designs of these sources differ substantially, they can be categorized by the physical principles that ultimately lead to extraction and ionization of analytes from a sample surface. The three main categories are spray-based, laser-based, and plasma-based methods.

Plasma-based devices are probably the most diverse group of ADI-MS sources because of the many types of discharges that have been used. Examples include direct- 
current plasmas [5-7], dielectric-barrier discharges (DBDs) $[8,9]$, radiofrequency glow discharges [10], and microhollow cathode discharges [11]. It is commonly believed that most of the plasma-based ADI-MS sources thermally desorb analytes and ionize them with reagent ions, similarly to APCI, that are generated from the interaction of plasma species with atmospheric constituents. While many of these plasmas have been fundamentally characterized, it is often in the context of their use in atomic spectroscopy. In such studies, these discharges have been found to differ drastically in electron number density $\left(\mathrm{n}_{\mathrm{e}}\right)$, excitation temperature $\left(\mathrm{T}_{\mathrm{exc}}\right)$, gas-kinetic temperature, and other conventional plasma parameters. Although these factors are known to dictate the performance of a plasma in atomic analyses, their role in ionization of molecules for mass spectrometry is not necessarily the same.

A few publications have addressed fundamental characteristics of discharges utilized for molecular mass spectrometry. A transient microenvironment mechanism was used to explain analyte ionization and matrix effects observed with direct analysis in real time (DART), a DC-discharge-based source [12]. Similarly, ionization matrix effects for three plasma-based sources, DART, the flowing atmospheric-pressure afterglow (FAPA), and the low-temperature plasma (LTP) probe, were compared based on analyte and matrix proton affinity [13]. Ionization mechanisms for DART in the negativeionization mode were compared with those for APCI and atmospheric-pressure photoionization (APPI) by analyzing a large suite of molecules [14, 15]. Franzke et al. [16] compared emission spectra from a DBD, using different discharge gases, with protonated analyte signals from an ion-mobility spectrometer.

Our research group has examined in some detail three plasma-based ADI-MS sources. Direct-current, helium discharges used by DART and FAPA were examined by optical and mass spectrometry to characterize discharge structures [17]. Spatially resolved emission from the DBD-based LTP probe was used to determine the origin of species such as water and oxygen that are important in the ionization process, and also conditions that affect formation of plasma species relevant to ADI-MS [18]. In a subsequent publication, these spatial profiles in conjunction with a detailed literature survey revealed that $\mathrm{He}_{2}^{+}$contributes significantly to the reagent-ion formation process [19]. Fundamental characterization of plasma-based ambient-ionization sources through optical and mass spectrometric means can yield insight into reactions taking place in the discharge and conditions affecting analyte desorption/ionization

In the present study, the atmospheric-pressure glow discharge (APGD) and the corresponding afterglow of the FAPA source were studied via optical spectroscopy under a variety of plasma operating conditions. Spatially resolved emission spectra from the discharge provide information about the species created in the plasma, as well as values for gas temperature and electron number density. These findings will be discussed in the context of ADI-MS analyses with the FAPA.

\section{Experimental}

\section{FAPA Source}

To enable emission from the discharge and afterglow of the FAPA to be detected, a special FAPA cell with a quartz body was constructed [17]. The helium APGD was established between a stainless-steel pin (3.1-mm diameter that tapers to a point with $\sim 39^{\circ}$ taper angle) and a brass plate that were sealed to a quartz tube (13.2-mm o.d., 12-mm i.d.) with Viton o-rings (cf. Figure 1). The distance between the electrodes was fixed at $7.5 \mathrm{~mm}$. A $1.6-\mathrm{mm}$ diameter hole in the center of the brass plate allowed species generated in the discharge to flow into the open atmosphere, resulting in the afterglow. A small raised edge around the hole in the plate inside the discharge chamber ensured that the discharge reproducibly formed from the pin to the raised edge on the hole. The discharge was always operated in a currentcontrolled mode with either a positive or negative potential applied to the pin electrode with a high-voltage, DC power supply (model BRC-5-400R; Universal Voltronics Corp., Mt. Kisco, NY, USA), while the plate electrode was always

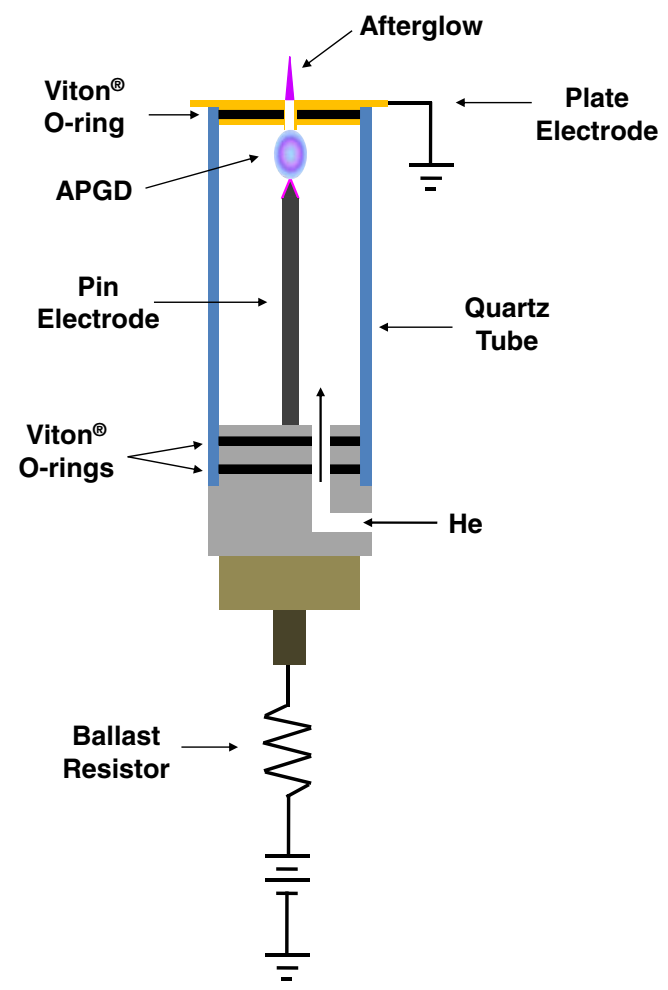

Figure 1. Diagram of the quartz-bodied FAPA cell used for the present optical studies. A positive or negative DC potential was applied to the pin electrode, while the brass plate was grounded. The inter-electrode distance was fixed at $7.5 \mathrm{~mm}$ for all experiments 
maintained at ground potential. A $5 \mathrm{k} \Omega$ ballast resistor was placed in series with the pin to compensate for the inherent negative dynamic resistance of glow discharges at atmospheric pressure. Ultrahigh purity helium (99.999\% purity; Airgas, Radnor, PA, USA) was supplied to the discharge chamber with a mass flow controller (MKS Instruments, Andover, MA, USA).

\section{Optical Emission Measurements}

Spatially resolved spectroscopic measurements were made with a commercial emission spectrometer (ACTIVA; Horiba-Jobin Yvon, Longjumeau, France) ordinarily used for inductively coupled plasma (ICP) atomic emission spectroscopy with a few optical modifications [20]. The quartz FAPA cell was positioned in place of the ICP torch on a translational stage for alignment with the slit of the spectrometer. The ACTIVA spectrometer utilizes a CzernyTurner configuration, which preserves the spatial image focused onto the entrance slit. With the aid of a twodimensional charge-coupled device (CCD) detector, spatial and spectral information were simultaneously obtained.

The image of the FAPA was demagnified by a ratio of 3.7:1, which resulted in a maximum observable distance of $25.6 \mathrm{~mm}$. This large viewing range enabled imaging of the APGD and the afterglow of the FAPA without changing the position of the source. However, the intensity of those two discharge regions was drastically different. Accordingly, to prevent detector saturation and blooming when emission from the weak afterglow was being measured, black paper was placed on the outside of the glass discharge cell to block the intense emission of the APGD. Integration times for the APGD and the afterglow were 1 to $10 \mathrm{~s}$ and $65 \mathrm{~s}$, respectively.

The entrance slit of the spectrometer was set to $10 \mu \mathrm{m}$, which led to a spectral resolution between 8 and $16 \mathrm{pm}$ for UV and visible wavelengths, respectively. This resolution was satisfactory even for distinguishing between different rotational features of molecules. The physical size of the APGD and the afterglow are narrow, less than $1.5 \mathrm{~mm}$ in diameter, so alignment of the source with respect to the spectrometer was crucial. This alignment was achieved with the spectrometer set to zero order; the source position was then adjusted for maximum signal. This maximum signal was checked every few hours and at the beginning of each day. Conveniently, the discharge was so stable that zeroorder emission never deviated more than $10 \%$ from the optimal signal, so the FAPA never needed to be realigned.

\section{Results and Discussion}

\section{Plasma Species Present in the APGD and Afterglow of the FAPA}

UV-visible emission spectra can help identify species present in a plasma that might be useful for plasma diagnostics and for characterizing ionization pathways. Andrade et al. [21] presented an emission spectrum from a He APGD in a sealed discharge chamber. In addition to the expected emission lines from $\mathrm{He}$, atomic emission from oxygen and nitrogen as well as emission bands of various diatomic species ( $\mathrm{NO}, \mathrm{OH}, \mathrm{N}_{2}$, and $\mathrm{N}_{2}{ }^{+}$) were observed. For a DC He APGD similar to that employed in FAPA, Gielniak et al. [22] also discovered the presence of $\mathrm{CN}$ and $\mathrm{NH}$. In both cases, the presence of non-helium species was attributed to impurities in the gas supply or transfer lines.

Table 1 lists the most abundant atomic and molecular species detected in the APGD of the FAPA used in the present study with a pin-negative configuration at $25 \mathrm{~mA}$ and a $\mathrm{He}$ flow rate of $1.5 \mathrm{~L} / \mathrm{min}$, which are the normal operating conditions of this source in ADI-MS analyses. Compared with the previously mentioned studies, only a few different species were detected in our APGD. Both atomic and molecular carbons, in the form of $\mathrm{CO}^{+}$, were readily observed in the lowwavelength region of the spectrum. The source of carbon in the discharge is believed to be the Viton o-ring used to seal the plate electrode to the inside of the glass chamber. As the plate electrode is heated by the discharge, the o-ring will outgas or, under extreme operating conditions, even melt, leading to the presence of hydrocarbons in the discharge.

Another species that was detected in the present APGD was the helium excimer, $\mathrm{He}_{2}{ }^{*}$, with multiple bands between 460 and $650 \mathrm{~nm}$ (cf. Table 1). The $\mathrm{He}_{2}{ }^{*}$ molecule is particularly interesting because it has a ground vibrational state of $\sim 18 \mathrm{eV}$ with respect to a ground state helium atom [23] and the upper energy levels of the observed transitions are greater than $20 \mathrm{eV}$ [24]; these energies are sufficient to cause Penning ionization of atmospheric constituents. Of particular interest are $\mathrm{N}_{2}$ and $\mathrm{H}_{2} \mathrm{O}$, with ionization energies of

Table 1. Major Atomic and Molecular Species Observed in the Emission Spectrum of the Helium APGD from the FAPA Operated at $25 \mathrm{~mA}$ with a Helium Flow Rate of $1.5 \mathrm{~L} / \mathrm{min}$

\begin{tabular}{|c|c|c|}
\hline $\begin{array}{l}\text { Emitting } \\
\text { species }\end{array}$ & Transition & Wavelength/nm \\
\hline NO & $\mathrm{A}^{2} \Sigma^{+}-\mathrm{X}^{2} \Pi$ & $202-273\left(v^{\prime}=0,1\right)$ \\
\hline $\mathrm{CO}^{+}$ & $\mathrm{B}^{2} \Sigma-\mathrm{X}^{2} \Sigma$ & $203-277\left(v^{\prime}=0,1,2,3,4,5\right)$ \\
\hline $\mathrm{OH}$ & $\mathrm{A}^{2} \Sigma^{+}-\mathrm{X}^{2} \Pi$ & $281-309\left(v^{\prime}=0,1\right)$ \\
\hline $\mathrm{N}_{2}$ & $\mathrm{C}^{3} \Pi_{\mathrm{u}}-\mathrm{B}{ }^{3} \Pi_{\mathrm{g}}$ & $295-434.5\left(v^{\prime}=0,1,2\right)$ \\
\hline $\mathrm{N}_{2}^{+}$ & $\mathrm{B}^{2} \Sigma_{\mathrm{u}}^{+}-\mathrm{X}^{2} \Sigma_{\mathrm{g}}^{+}$ & $387-471\left(v^{\prime}=0\right)$ \\
\hline \multirow[t]{4}{*}{$\mathrm{He}_{2}$} & $\mathrm{e}^{3} \Pi_{\mathrm{g}}-\mathrm{a}^{3} \Sigma_{\mathrm{u}}^{+^{+}}$ & $460-475\left(v^{\prime}=0\right)$ \\
\hline & $\mathrm{f}^{3} \Delta_{\mathrm{u}}^{\mathrm{g}}-\mathrm{b}{ }^{3} \Pi_{\mathrm{g}}$ & $568-605\left(v^{\prime}=0\right)$ \\
\hline & $\mathrm{F}^{1} \Delta_{\mathrm{u}}-\mathrm{B}{ }^{1} \Pi_{\mathrm{g}}$ & $605-630\left(v^{\prime}=0\right)$ \\
\hline & $\mathrm{d}^{3} \Sigma_{\mathrm{u}}^{+}-\mathrm{b}{ }^{3} \Pi_{\mathrm{g}}$ & $630-650\left(v^{\prime}=0\right)$ \\
\hline \multirow[t]{2}{*}{ H I } & $\mathrm{H}_{\beta}$ & 486.13 \\
\hline & $\mathrm{H}_{\alpha}$ & 656.28 \\
\hline \multirow[t]{6}{*}{ He I } & $3 \mathrm{p}^{3} \mathrm{P}_{2}{ }^{\circ}-2 \mathrm{~s}{ }^{3} \mathrm{~S}_{1}$ & 388.86 \\
\hline & $3 p^{1} \mathrm{P}_{1}{ }^{\circ}-2 \mathrm{~s}^{1} \mathrm{~S}_{0}$ & 501.57 \\
\hline & $3 d^{3} D_{1,2,3}-2 p{ }^{3} P_{0,1,2}{ }^{0}$ & 587.56 \\
\hline & $3 \mathrm{~d}^{1} \mathrm{D}_{2}-2 \mathrm{p}{ }^{1} \mathrm{P}_{1}{ }^{\circ}$ & 667.82 \\
\hline & $3 s^{3} \mathrm{~S}_{1}-2 \mathrm{p}{ }^{3} \mathrm{P}_{0,1,2}$ & 706.52 \\
\hline & $3 \mathrm{~s}^{1} \mathrm{~S}_{0}-2 \mathrm{p}^{1} \mathrm{P}_{1}{ }^{\circ}$ & 728.14 \\
\hline \multirow[t]{3}{*}{ O I } & $\left({ }^{4} \mathrm{~S}^{\circ}\right) 3 \mathrm{p}{ }^{5} \mathrm{P}_{3}-\left({ }^{4} \mathrm{~S}^{\circ}\right) 3 \mathrm{~s}{ }^{5} \mathrm{~S}_{2}{ }^{\circ}$ & 777.194 \\
\hline & $\left({ }^{4} \mathrm{~S}^{\circ}\right) 3 \mathrm{p}{ }^{5} \mathrm{P}_{2}-\left({ }^{4} \mathrm{~S}^{\circ}\right) 3 \mathrm{~s}^{5} \mathrm{~S}_{2}{ }^{\circ}$ & 777.417 \\
\hline & $\left({ }^{4} \mathrm{~S}^{\circ}\right) 3 \mathrm{p}{ }^{5} \mathrm{P}_{1}-\left({ }^{4} \mathrm{~S}^{\circ}\right) 3 \mathrm{~s}^{5} \mathrm{~S}_{2}{ }^{\circ}$ & 777.539 \\
\hline
\end{tabular}


15.6 and $12.6 \mathrm{eV}$, respectively [25], because the ionic forms of these molecules are well known reagent species in APCI; these reagent ions can undergo charge-transfer or proton-transfer ionization with gas-phase analyte molecules, leading to simple mass spectra and a wide-range of ionizable analytes.

It was earlier theorized that reagent ions in helium-plasma ambient ionization sources were produced by Penning ionization of $\mathrm{N}_{2}$ and $\mathrm{H}_{2} \mathrm{O}$ with helium metastable atoms, $\mathrm{He}^{\mathrm{m}}[6,8,10]$. However, it was recently demonstrated with the LTP probe that at least $30 \%$ of $\mathrm{N}_{2}{ }^{+}$is formed through charge transfer with $\mathrm{He}_{2}{ }^{+}$[19]. Furthermore, a theoretical characterization of a helium APGD [26] found that the number densities of $\mathrm{He}^{\mathrm{m}}$ and $\mathrm{He}_{2}{ }^{*}$ in the bulk of the plasma were approximately the same. Therefore, it is reasonable to hypothesize that $\mathrm{He}_{2}{ }^{*}$ serves as an additional energy carrier from the discharge to the atmosphere and would, thus, be an additional mechanism of reagent-ion formation. This mechanism might be particularly relevant for DART, in which grids are placed after the discharge region such that only neutral species enter the open atmosphere.

In contrast to the APGD itself, the UV-visible emission spectrum of the afterglow was much weaker and simpler (cf. Figure S-1). Because emission from the afterglow is very weak under usual FAPA operating conditions, $1.5 \mathrm{~L} / \mathrm{min}$ helium and $25 \mathrm{~mA}$ discharge current, the gas flow rate, and discharge current were increased to $3.25 \mathrm{~L} / \mathrm{min}$ and $50 \mathrm{~mA}$, respectively, to produce the spectrum in Figure S-1. Additionally, the integration time of the detector was set to the maximum, $65 \mathrm{~s}$. The only detected atomic species were hydrogen and oxygen, likely from the dissociation of $\mathrm{H}_{2} \mathrm{O}$ and $\mathrm{O}_{2}$, respectively [18], with the rest of the spectrum consisting of vibrational bands of molecules. The strongest emitting species were $\mathrm{OH}\left(\mathrm{A}^{2} \Sigma^{+}\right)$and $\mathrm{N}_{2}^{+}\left(\mathrm{B}^{2} \Sigma_{\mathrm{u}}^{+}\right)$, which were significantly more intense than any other species in the afterglow. Both $\mathrm{OH}$ and $\mathrm{N}_{2}{ }^{+}$are thought to be important in the formation of protonated water clusters, $\left(\mathrm{H}_{2} \mathrm{O}\right)_{n} \mathrm{H}^{+}$, which are the most abundant positive reagent ions produced by the FAPA [5]. Other molecular species found in the afterglow spectrum include $\mathrm{CO}^{+}\left(\mathrm{B}^{2} \Sigma\right)$ and $\mathrm{NH}\left(\mathrm{A}^{3} \Pi\right)$. Surprisingly, no emission was detected from any helium species, indicating that the majority of excited helium species in the afterglow have either decayed to a state that emits outside of the accessible spectral window (e.g., $\mathrm{He}^{*}$ with $\mathrm{n}=2$ or $\mathrm{He}^{\mathrm{m}}$ ), or exist in a form that does not decay radiatively (e.g., $\mathrm{He}_{2}{ }^{+}$).

\section{Effect of He Flow Rate and Discharge Polarity on Emitting Species in the He APGD}

Spatially resolved profiles of emitting species in atmosphericpressure discharges have already provided information on the source of impurities in the discharge, the relative distribution of ions and excited species needed for analyte excitation/ ionization, and even mechanisms of ion formation [18, 19]. Here, the polarity, operating current, and gas flow rate of the FAPA were varied to determine the discharge structure and conditions that favor production of species essential for APCI ionization pathways. Figure $2 \mathrm{a}$ and $\mathrm{b}$ show the spatial profile of the He I line at $706.5 \mathrm{~nm}$ for two He APGDs in a pin-negative and a pin-positive configuration, respectively. For all APGD profiles, a discharge distance (x-axis) of zero corresponds to the tip of the pin electrode, with positive distances approaching the plate electrode. Optimal mass spectrometric performance of the FAPA typically arises at $25 \mathrm{~mA}$ in the pin-negative polarity [27]. As a comparison, the $2 \mathrm{~mA}$ discharge is more similar to the one used in the DART source [17, 28].

Both polarities and current levels exhibited distinct emission maxima at both the cathode and the anode. For the $25-\mathrm{mA}$ discharge in the pin-negative configuration (cf. Figure 2a, blue trace), the He I maximum resides on the cathode pin and is due to the strong emission from the negative glow, which is where the majority of helium ions and excited species are produced. In addition, the emission (a)

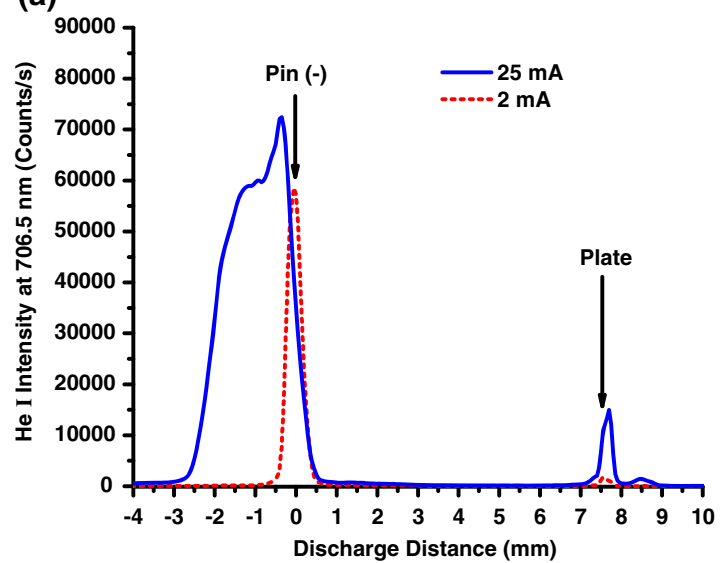

(b)

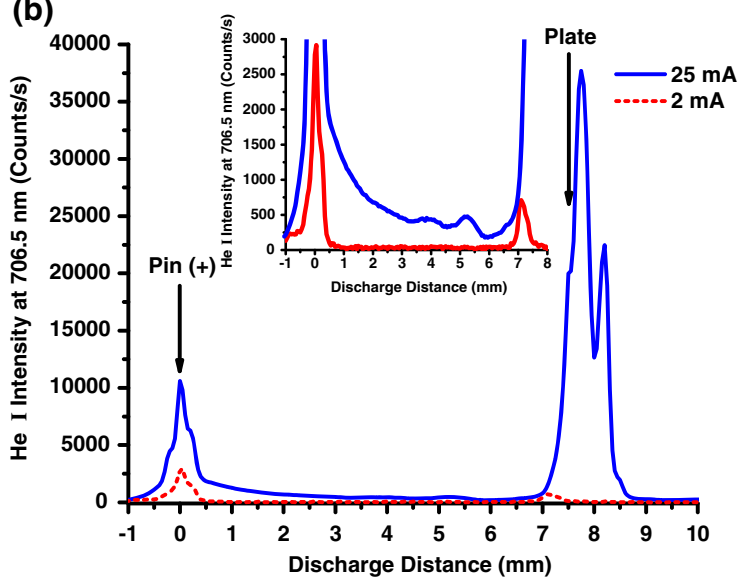

Figure 2. Spatially resolved emission profiles of the 706.5-nm He I line in the APGD at discharge currents of $25 \mathrm{~mA}$ (blue trace) and $2 \mathrm{~mA}$ (red trace) with a helium flow rate of $1.5 \mathrm{~L} / \mathrm{min}$. The discharge was operated in both the pin-negative (a) and pinpositive (b) polarity. The inset in (b) is on an expanded vertical axis to demonstrate helium emission in the positive column of the 25-mA APGD. In all APGD plots, a discharge distance of zero refers to the tip of the pin electrode 
intensity for He I was distributed along the length of the pin $(\sim 2 \mathrm{~mm})$ and throughout the positive column. For the pinnegative, 2-mA discharge (cf. Figure 2a, red trace), the Heemission maximum was located at the very tip of the pin and was shifted $300 \mu \mathrm{m}$ from that of the $25-\mathrm{mA}$ discharge. Even though the operating current differs by only one order of magnitude, the locations of the emission maxima indicate an altered plasma structure; specifically, the 2-mA discharge exhibits corona-to-glow discharge behavior. In the case of a corona discharge, the electric field decays exponentially from the needle electrode, with the ionic current density residing almost exclusively at the tip of the pin, regardless of polarity. Conversely, in an atmospheric-pressure glow discharge the strongest electric field and highest current density reside in the negative glow near the cathode; however a substantial electric field and current density exist between the two electrodes in the positive column.

Although the maximum emission from the two current levels was similar, the $\mathrm{He} \mathrm{I}$ intensity for the $25-\mathrm{mA}$ discharge integrated along the entire plasma was more than five times that of the 2-mA discharge. The greater abundance of excited $\mathrm{He}$ created in the 25-mA APGD should lead to more efficient production of $\mathrm{N}_{2}{ }^{+}$and a greater density of reagent ions. In fact, the $\mathrm{N}_{2}{ }^{+}$-produced has been mass spectrometrically and optically shown to be much higher, at least 17 times more abundant, with the FAPA plasma than with the 2-mA discharge [17]. Both current levels exhibited a second maximum in emission intensity at the anode, where electric field and electron energies should be low. Models of a helium APGD similarly showed a second maximum in number density at the anode for nearly all species included in the model [26]. Unlike in reducedpressure GDs, a positive potential gradient in the positive column towards the anode causes an excess of low-energy electrons at the anode, which are capable of ionizing/ exciting plasma species [26]; the high electron densities at the anode are likely the origin of the second emission maximum.

(a)

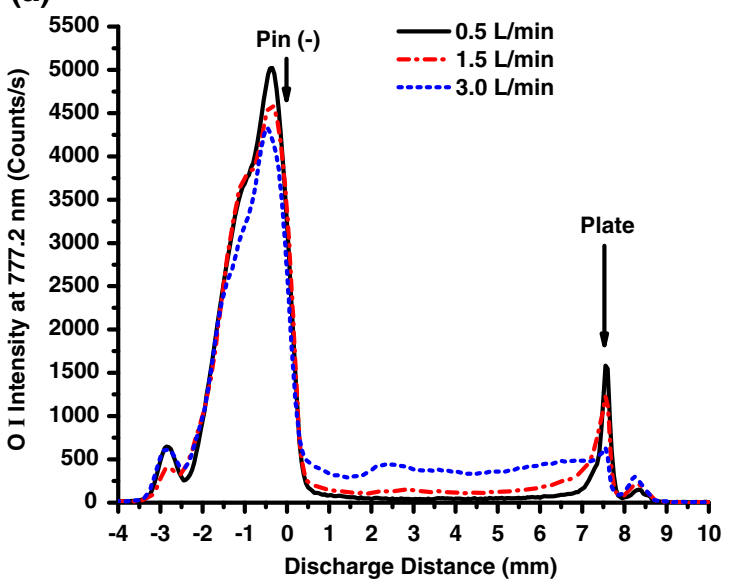

When the polarity of the $25-\mathrm{mA}$ discharge was reversed to the pin-positive mode (cf. Figure 2b, blue trace), maximum He I emission occurred at the plate cathode where the negative glow resides. The relative amount of excited helium in the positive column was higher with this polarity. In pin-positive mode, the area of the negative glow is restricted to the surface of the raised edge on the exit plate, which causes a larger portion of the current density to exist in the positive column. It would at first seem that this polarity would be favored for ambient MS analyses because the negative glow and, thus, the greatest source of He*, would be closer to the sample. However, the integrated $\mathrm{He}$ emission for the pin-negative polarity was more than four times greater than that for the pin-positive configuration with an operating current of $25 \mathrm{~mA}$. While the cause for the differences in emission intensities is not yet known, this result supports the finding that the FAPA performs better as an ionization source with a pin cathode [5, 27].

When the pin-positive discharge was operated at $2 \mathrm{~mA}$, the strongest helium emission still existed at the tip of the pin (cf. Figure 2b, red trace). As the inset in Figure 2b shows, excited helium was observed between the two electrodes at $25 \mathrm{~mA}$, whereas no such emission could be detected with the discharge operated at $2 \mathrm{~mA}$. Similar behavior at these two operating currents was observed for $\mathrm{N}_{2}{ }^{*}(337 \mathrm{~nm})$ and $\mathrm{O} \mathrm{I}(777 \mathrm{~nm})$ emission. These data provide further evidence that the discharge operated at $2 \mathrm{~mA}$ behaves like a corona rather than a true glow such as the FAPA maintained at $25 \mathrm{~mA}[17,21]$.

Several species from impurities were also detected in the APGD of the FAPA at various plasma-gas flow rates and for both polarities. Emission profiles of atomic oxygen at $777 \mathrm{~nm}$ in a pin-negative and a pin-positive APGD are shown in Figure 3a and b, respectively. The presence and location of atomic oxygen is particularly important for mass spectrometric analyses. It has been noted in APCI reactions that the neutral atomic oxygen radical can react with aromatic bonds to form alcohols [29, 30]. This reaction

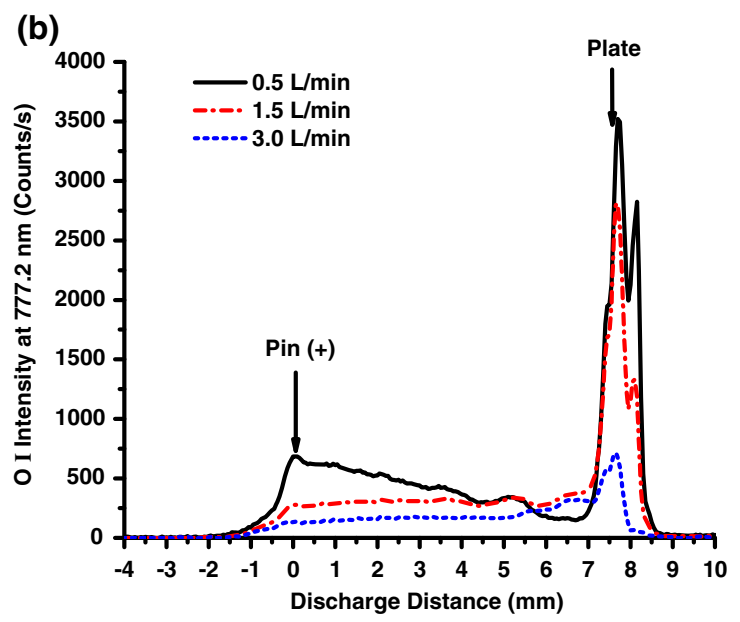

Figure 3. Atomic oxygen emission profiles $(777 \mathrm{~nm})$ for the APGD operated in the pin-negative (a) and pin-positive (b) configuration for a variety of He plasma-gas flow rates 
often results in complicated mass spectra for aromatic molecules, which feature not only molecular ion peaks but also a series of oxygen-adduct peaks [17]. As a result, determining the source of oxygen and factors that limit atomic oxygen production is of importance for these sources. For both discharge polarities, O I emission near the plate region (i.e., closest to the open atmosphere) declines with increasing He flow rate. This finding suggests that an appreciable amount of atmospheric oxygen is diffusing into the discharge region through the hole in the plate electrode. Higher helium flows oppose diffusion into the discharge and thereby reduce the amount of oxygen reaching the negative glow region. This finding is congruous with spectroscopic studies on a similar atmospheric-pressure, helium discharge, the LTP probe, which showed that the majority of oxygen in the discharge region was due to diffusion from the atmosphere [18].

This trend does not necessarily hold for other regions of the APGD, particularly for the positive column. We attribute these differences to the concentration gradient of impurities near the plate electrode. As atmospheric constituents diffuse through the hole into the discharge chamber, their volume expands as the linear velocity of the plasma gas drops. This effect results in a lower impurity concentration in the discharge chamber than in the narrow channel separating the discharge from the atmosphere. As a result, diffusion and plasma-gas flow rate would contribute minimally to variances in impurity concentration within the larger volume of the discharge chamber. Changes in flow rate would alter only the amount of reactive species formed in these regions.

In fact, the oxygen emission in the positive column monotonically increased with flow rate for the pin-negative configuration, while it had the opposite effect for the pinpositive configuration. This difference is likely due to the different reaction pathways occurring at the cathode and the anode. Theoretical modeling has suggested that at the cathode, the majority of $\mathrm{He}^{+}$is produced from electron impact with ground state helium [26]. Because the greatest potential drop is in the cathode dark space, the electrons in this region have sufficient energy to ionize ground-state helium directly. In contrast, and as was mentioned earlier, low-energy electrons accumulate at the anode because of the slight potential gradient in the positive column. Because electron energies are so low in this region, $\mathrm{He}^{+}$is almost exclusively produced from electron impact with previously formed $\mathrm{He}^{*}$ [26]. From these factors, more $\mathrm{He}_{2}{ }^{+}$and other species important in the dissociation/ excitation process exist at the anode than other places in the APGD, which likely contributes to the enhanced sensitivity of the FAPA in the pin-negative configuration.

The spatial distribution of two other species important in the ionization pathways of the FAPA source, $\mathrm{OH}(306.0 \mathrm{~nm})$ and $\mathrm{N}_{2}^{+}(391.4 \mathrm{~nm})$, are shown in Figure S-2a and S-2b, respectively. These profiles were collected under different gas flow rates for a pin-positive APGD. The $\mathrm{OH}$ emission dropped monotonically with helium flow rate across the entire APGD, suggesting that the majority of $\mathrm{OH}$ was produced from a species that diffused into the discharge from the atmosphere. This trend was observed for the opposite discharge polarity, as well (data not shown). For $\mathrm{N}_{2}{ }^{+}$(Figure S-2b), emission similarly declined with increasing He flow at both electrodes, further suggesting that most of the nitrogen in the discharge chamber was not from the gas supply or transfer lines. Interestingly, $\mathrm{N}_{2}{ }^{+}$emission near the plate electrode was stronger in the pin-negative configuration providing further explanation why better mass spectrometric performance is seen with the FAPA in this polarity. Regardless of discharge polarity, $\mathrm{N}_{2}{ }^{+}$had a strong emission maximum at the anode, which decayed deep into the positive column. In a helium APGD, the majority of $\mathrm{N}_{2}{ }^{+}$is formed from a chargetransfer reaction involving $\mathrm{He}_{2}{ }^{+}$[26]. Further, the $\mathrm{He}_{2}{ }^{+}$density is known to be the greatest at the anode and to slowly decay into the positive column, mirroring the $\mathrm{N}_{2}^{+}$emission profile. Therefore, we believe this feature is indicative of the presence of $\mathrm{He}_{2}{ }^{+}$.

\section{$\mathrm{N}_{2}{ }^{+}$Emission Profiles in the Afterglow}

Because emission from the afterglow of the FAPA was very weak, spatially resolved spectral information could be collected for only two emitting species, $\mathrm{N}_{2}{ }^{+}$and $\mathrm{OH}$. Additionally, the spatial emission behavior of both molecules was nearly identical, so only $\mathrm{N}_{2}^{+}$will be discussed here. Afterglow emission profiles for the $\mathrm{N}_{2}{ }^{+}$bandhead at $391.4 \mathrm{~nm}$ for various discharge currents and helium flow rates are shown in Figure $4 \mathrm{a}$ and $\mathrm{b}$, respectively. An afterglow distance of zero in these profiles corresponds to the edge of the plate electrode, with positive values extending into the open atmosphere. In Figure 4a the APGD current was incremented from $30 \mathrm{~mA}$ to $70 \mathrm{~mA}$ at a fixed $\mathrm{He}$ flow rate of $2.9 \mathrm{~L} / \mathrm{min}$. Emission of $\mathrm{N}_{2}^{+}$in the afterglow became stronger at higher operating currents, because of the greater production of reactive species within the discharge that are capable of ionizing and exciting $\mathrm{N}_{2}$. The $\mathrm{N}_{2}{ }^{+}$ emission decayed to baseline approximately $4 \mathrm{~mm}$ from the plate electrode, regardless of discharge current. The presence of $\mathrm{N}_{2}{ }^{+}$emission in the afterglow is therefore likely dependent on diffusion of an atmospheric quenching agent, which then undergoes charge transfer.

This hypothesis is supported by the behavior of afterglow emission profiles at changing He flow rates (cf. Figure 4b). Both the amount of $\mathrm{N}_{2}{ }^{+}$generated and the location where emission decayed to baseline increased monotonically with He flow rate. At higher flows, more excited/ionized helium species are generated per unit time and, at the same time, the outward gas velocity opposes the inward diffusion of quenchers into the beam. This behavior in the afterglow is the opposite of what was observed in the APGD, where $\mathrm{N}_{2}{ }^{+}$ emission decreased with plasma-gas flow rate. We attribute this disparity to the relative concentrations of reactants leading to $\mathrm{N}_{2}^{+}$formation. In the APGD, $\mathrm{N}_{2}$ is limiting as it must diffuse from the atmosphere into the discharge chamber through the hole in the plate electrode, through 
(a)

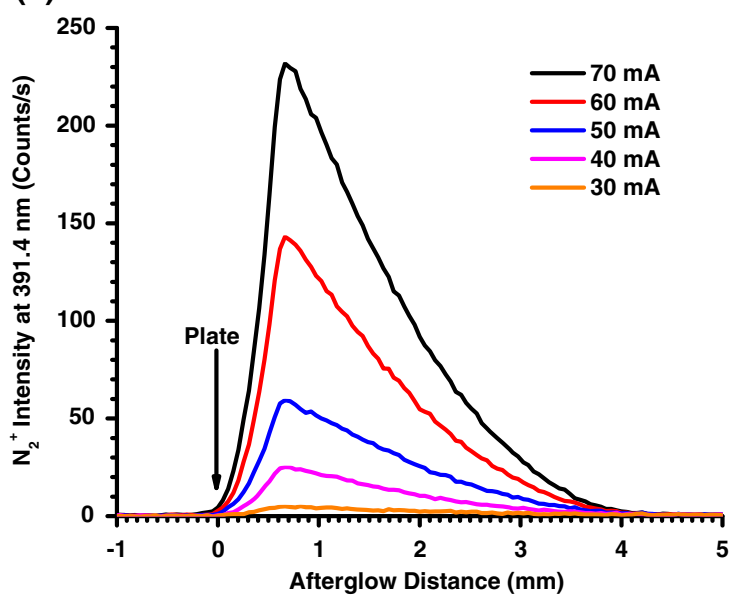

(b)

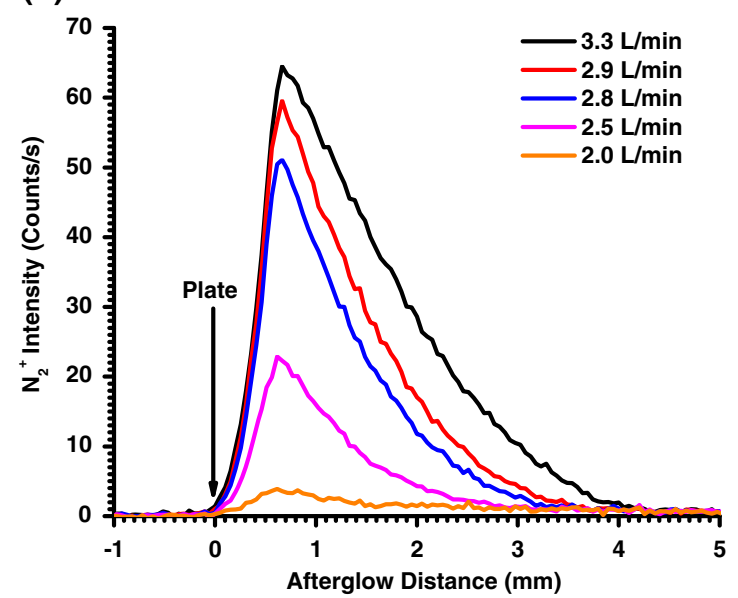

Figure 4. Afterglow emission of the $\mathrm{N}_{2}{ }^{+}$bandhead at $391.4 \mathrm{~nm}$ for a pin-negative FAPA. The traces in (a) are for different discharge currents at a $\mathrm{He}$ flow rate of $2.9 \mathrm{~L} / \mathrm{min}$, whereas (b) demonstrates the effect of He flow rate on $\mathrm{N}_{2}{ }^{+}$emission at a discharge current of $50 \mathrm{~mA}$. In both (a) and (b), an afterglow distance of zero refers to the edge of the plate electrode, with positive values extending into the open atmosphere

which a high opposing velocity of plasma gas issues; in the afterglow, the velocity of the discharge gas is lower than in the hole of the plate electrode, resulting in a greater concentration of diffused $\mathrm{N}_{2}$ in the afterglow and making excited helium species the limiting reactants.

Another interesting feature of the afterglow emission profiles is that the maximum occurred consistently about $0.67 \mathrm{~mm}$ downstream from the anode plate. Because the emission maximum of $\mathrm{OH}$ and $\mathrm{N}_{2}{ }^{+}$did not occur immediately at the exit of the plate and because the afterglow is effectively a field-free region, it is likely that some species are acting as a carrier to transfer energy from the discharge into the open atmosphere, where no external source of energy exists, leading to the ionization of nitrogen and dissociation of water vapor. Potential plasma species that could serve as energy carriers to the afterglow will be discussed later in this paper.

\section{OH Rotational Temperatures and Electron Number Densities of the APGD}

The rotational temperature $\left(\mathrm{T}_{\text {rot }}\right)$ is an important diagnostic for plasma-based ambient desorption/ionization sources because it approximates the gas-kinetic temperature, which correlates with desorption of molecules from surfaces for many plasmabased ADI-MS sources [2, 31]. Rotational temperatures are derived from the Boltzmann population distribution among rotational energy levels of a well characterized thermometric molecule. Though numerous diatomics have been successfully used to measure $\mathrm{T}_{\text {rot}}$, only $\mathrm{OH}$ and $\mathrm{N}_{2}{ }^{+}$offered sufficient intensity in the APGD and afterglow of the FAPA to be useful in the present study (cf. Figure S-1). Because of non-thermal excitation from excited helium species, $\mathrm{N}_{2}^{+}$does not yield reliable gas-kinetic temperature values [19]; this fact will be demonstrated below for the afterglow region. In the case of the
FAPA, only $\mathrm{OH}$ temperatures should be regarded as being equivalent to gas-kinetic temperature.

Four emission lines in the $\mathrm{Q}_{1}$ branch $[32,33]$ were used for determining $\mathrm{OH}$ rotational temperatures as they had sufficient intensity. These lines were the same as those used by Chan et al. [18] Plotting the unit-less quantity $\ln (I \lambda / g A)$ versus $E_{e x c}$, where $I$ is intensity of the line, $\lambda$ is the emission wavelength, $A$ is the transition probability, $g$ is the degeneracy of the excited energy level, and $E_{e x c}$ is the energy of the upper excited state in $\mathrm{eV}$, yields a linear plot with a slope equal to $\left(-1 / \mathrm{k} T_{\text {rot }}\right)$, where $\mathrm{k}$ is the Boltzmann constant in $\mathrm{eV} \mathrm{K}^{-1}$ and $\mathrm{T}_{\text {rot }}$ is the rotational temperature in $\mathrm{K}$ [34]. It should be noted that the Boltzmann distribution equation contains numerous constants and unit-conversion factors that would be included in the quantity $\ln (I \lambda / g A)$. However, due to the properties of logarithms, these constants will affect only the intercept of the plot and, thus, can be excluded when determining rotational temperature. Only temperature values where the correlation coefficient $\left(\mathrm{r}^{2}\right)$ of the fit was greater than 0.99 were used. The relative standard deviation of the measured temperature was taken as the relative error of the slope from the linear fit, which was less than $10 \%$ in the APGD.

The spatial profiles of $\mathrm{OH}$ rotational temperatures of the APGD for pin-negative and pin-positive modes are given in Figure $5 \mathrm{a}$ and $\mathrm{b}$, respectively. The maximum $\mathrm{T}_{\text {rot }}$ of $1170 \mathrm{~K}$ for the pin-negative configuration was located at the tip of the pin within the negative glow. The temperature throughout the positive column was lower and remained roughly constant at $\sim 770 \mathrm{~K}$. These temperatures are more than $600 \mathrm{~K}$ and $200 \mathrm{~K}$ lower than those measured for He APGDs with a restricted cathode and positive column [22] and sealed from air with a restricted cathode area [21], respectively. The reason for the drastic temperature differences could be due to the confined cathode area utilized in those APGDs, but this effect will be explored in the future. 
(a)

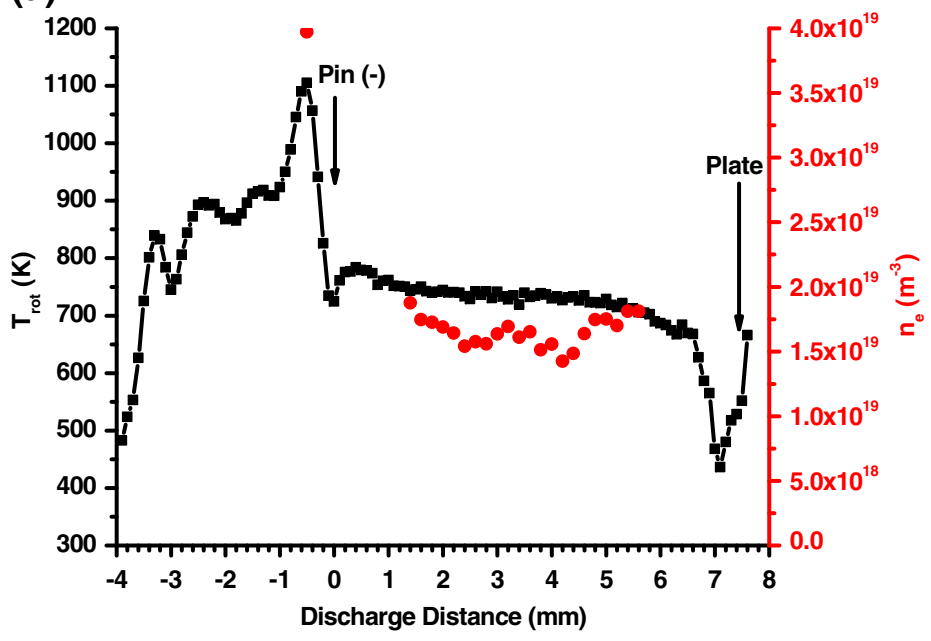

(b)

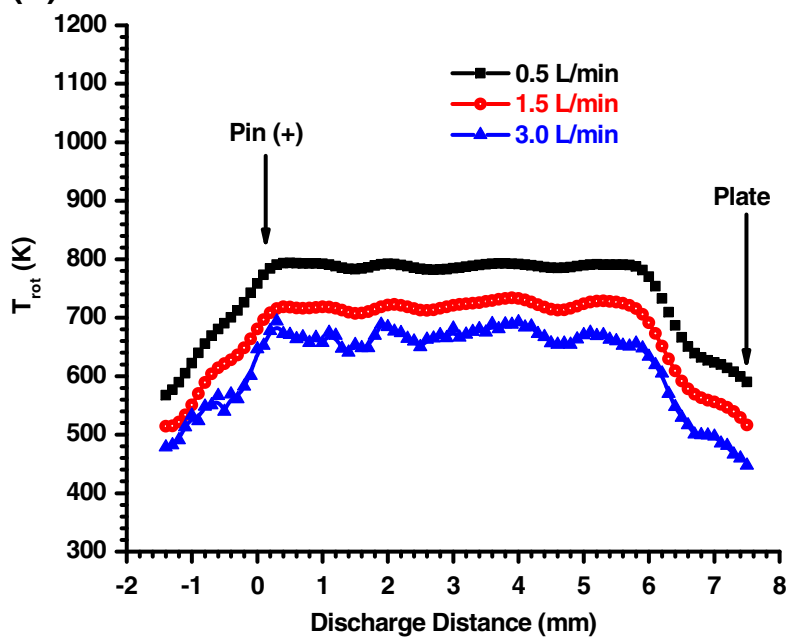

Figure 5. (a) $\mathrm{OH}$ rotational temperatures, $\mathrm{T}_{\text {rot }}$, and electron number densities, $\mathrm{n}_{\mathrm{e}}$, for a 25-mA, pin-negative APGD with a helium flow of $1.5 \mathrm{~L} / \mathrm{min}$. (b) $\mathrm{OH}$ rotational temperatures for a 25-mA, pin-positive APGD for selected gas flow rates. The relative error of all measurements was less than $10 \%$ and was assumed to be the same as those of the fits

When the polarity of the APGD was reversed (cf. Figure $5 \mathrm{~b}$ ), the temperature of the positive column was approximately the same as in the pin-negative discharge; however, the maximum $\mathrm{T}_{\text {rot }}$ was much lower, $780 \mathrm{~K}$, and was located in the positive column. Raising the plasma-gas flow rate for either polarity resulted in a drop in temperature, likely due to convective heat transfer. Ultimately, the lower maximum temperature in the pin-positive mode would result in less efficient desorption and could be one of the reasons the FAPA offers less sensitivity in the pin-positive polarity.

Spatially resolved electron number densities, $\mathrm{n}_{\mathrm{e}}$, in the pinnegative APGD were measured from the Stark broadening of the hydrogen beta line, $\mathrm{H}_{\beta}$, at $486.1 \mathrm{~nm}$. This spectral line is preferred because its broadening parameters are well known and are less affected by perturbers other than electrons $[35,36]$.

In addition to Stark broadening, the measured $\mathrm{H}_{\beta}$ line for this type of system is known to experience appreciable contributions from instrumental and van der Waals broadening. The $\mathrm{H}_{\beta}$ line in the positive column was fit with a Voigt profile containing a fixed Gaussian component of $13.7 \mathrm{pm}$, which was previously found to be the instrumental broadening of this particular spectrometer [18]. The Lorentzian contribution from van der Waals broadening was determined from the LindholmFoley impact theory $[37,38]$ to account for changes in local temperature with the spatially resolved $\mathrm{T}_{\text {rot }}$ shown in Figure $5 \mathrm{a}$. The van der Waals width was subtracted from the Lorentzian component of the Voigt fit to yield an estimated Stark width. Electron number density was calculated from the formula provided by Gigosos et al. [39] The relative error of $n_{e}$ was taken as the relative error of the Lorentzian width from the fit and was less than $15 \%$.

Estimating electron number density in the negative-glow region near the pin was similar to the process described above but required an additional step. It is known that broadening of hydrogen emission lines can also be caused by strong external electric fields $[40,41]$. Glow discharges at atmospheric pressure have a very thin cathode dark space, due to the very short mean free path, where the majority of the potential drop occurs. The result is a very high electric field $(>10 \mathrm{kV} / \mathrm{cm})$ at the tip of the pin electrode, which introduces an additional broadening component. Thus, the $\mathrm{H}_{\beta}$ profiles near the pin cathode exhibited two distinct Lorentzian profiles; an intense, narrow component indicative of the true electron number density and a less intense, broad component due to the strong electric field. To deconvolute these two broadening contributions, the center narrow portion of the profile was removed, leaving the broad wings due solely to the electric field. The wings were fit with a Lorentzian, which was then subtracted from the original emission line. The remaining profile was then treated like those in the positive column.

The estimated $n_{e}$ values in the pin-negative APGD with 1.5 L/min helium flow rate are shown in Figure $5 \mathrm{a}$. The relative error of $n_{e}$ was taken as the relative error of the Lorentzian width from the fit and was less than $15 \%$. Because of the involved data processing needed to determine $\mathrm{n}_{\mathrm{e}}$ in the negative glow, only one data point is shown. The electron density reaches a maximum of $\sim 4 \times 10^{19} \mathrm{~m}^{-3}$ at the same location as the highest emission intensity and rotational temperature. The $\mathrm{n}_{\mathrm{e}}$ drops in the positive column to $\sim 1.5 \times 10^{19} \mathrm{~m}^{-3}$.

Compared with values derived from a numerical simulation of a helium APGD [26], the $n_{e}$ figures in this study are almost exactly one order of magnitude higher. The relative spatial distribution of $n_{e}$ in the APGD was nearly identical between the two studies. Unfortunately, $\mathrm{H}_{\beta}$ was too weak at the anode plate to measure electron number densities. Assuming the number of ions and electrons are equal at each location in the discharge, the degree of ionization within the APGD is on the order of $10^{-6}$, based on the ideal 
gas law. This value is fairly low, considering that reducedpressure GDs have ionization efficiencies between $10^{-5}$ and $10^{-3}[42,43]$. However, because the FAPA is at atmospheric pressure, the number of collisions per second is about three orders of magnitude higher than in a reduced-pressure GD, so ionization reaction rates should be comparable.

\section{Rotational Temperatures in the Afterglow}

The gas temperature in the afterglow region of the FAPA is particularly important because samples are introduced directly into the afterglow and thermal processes are believed to play a major role in the desorption of molecules from their surfaces. Infrared thermography has already been used to approximate the temperature of the FAPA gas stream impinging on a glass surface $[17,44]$, but it is unknown how much the surface affects the measured temperature. Therefore, $\mathrm{OH}$ rotational temperatures were spectroscopically determined here for the afterglow of a pin-negative FAPA operated at $70 \mathrm{~mA}$ with a $\mathrm{He}$ flow rate of $3.25 \mathrm{~L} / \mathrm{min}$ (cf. Figure 6). Within the error of the measurement, the $\mathrm{OH} \mathrm{T}_{\text {rot }}$ was constant at $\sim 500 \mathrm{~K}$ across the 3-mm range where temperature could be reliably measured. Although thermographic measurements of the FAPA afterglow temperature were not obtained with the present combination of relatively high operating currents and gas flow rates, the maximum temperature increases linearly with current, but falls with flow rates above $1.5 \mathrm{~L} / \mathrm{min}$. Extrapolating from the thermographic data in Ref. [41], the maximum temperature under the present conditions would be $500 \mathrm{~K}$, in excellent agreement with the spectroscopic values.

In addition to the $\mathrm{OH}$ rotational temperature, the $\mathrm{N}_{2}{ }^{+}$ emission profile and $\mathrm{N}_{2}{ }^{+}$rotational temperature are shown in Figure 6. The $\mathrm{T}_{\text {rot }}$ for $\mathrm{N}_{2}{ }^{+}$was determined in the same way as described by Chan et al. [19] with all Boltzmann plots

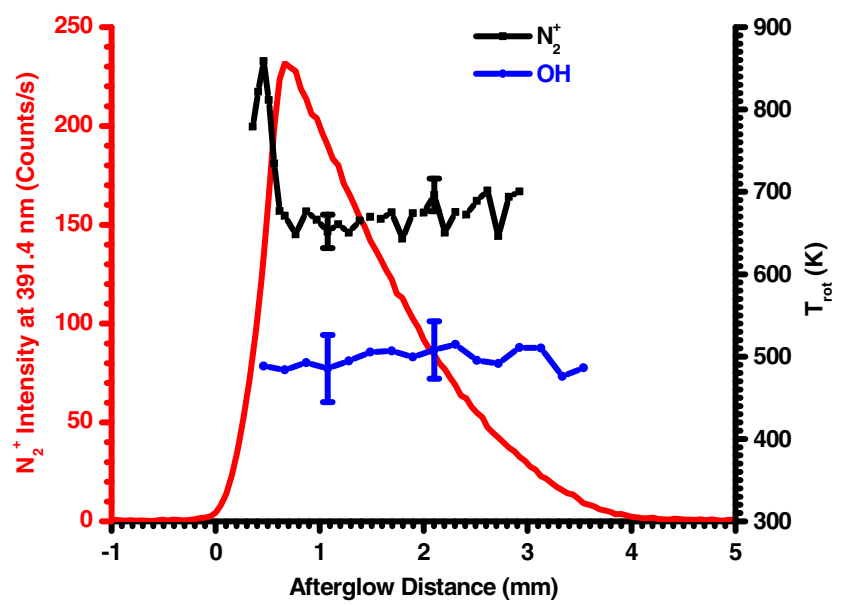

Figure 6. Rotational temperatures for $\mathrm{OH}$ (blue trace) and $\mathrm{N}_{2}{ }^{+}$(black trace) in the afterglow of the pin-negative FAPA at $3.25 \mathrm{~L} / \mathrm{min}$ and $70 \mathrm{~mA}$. The red trace shows the $\mathrm{N}_{2}{ }^{+}$emission profile as a frame of reference exhibiting good linearity $\left(\mathrm{r}^{2}>0.99\right)$. The $\mathrm{N}_{2}{ }^{+}$temperature reaches a maximum of $860 \mathrm{~K}$ and then drops to nearly a constant value of $\sim 670 \mathrm{~K}$ throughout the rest of the afterglow. Clearly, a discrepancy of $\sim 150 \mathrm{~K}$ exists between the temperature measurements from $\mathrm{OH}$ and $\mathrm{N}_{2}{ }^{+}$for this discharge. Because the measured $\mathrm{OH}$ temperature is similar to the thermographic temperatures, it is likely that the $\mathrm{N}_{2}{ }^{+}$ population distribution violates the assumption that a rapid equilibrium exists between the rotational and translational energies of the molecule. We have previously demonstrated that the $\mathrm{N}_{2}{ }^{+}$temperature is a good indicator of the dominant ionization mechanism of nitrogen [19]. In particular, charge transfer between $\mathrm{He}_{2}^{+}$and $\mathrm{N}_{2}$ yields a $\mathrm{N}_{2}^{+}$rotational temperature of $\sim 900 \mathrm{~K}$ [45], whereas charge transfer with $\mathrm{He}^{+}$and Penning ionization with $\mathrm{He}^{\mathrm{m}}$ produce nonlinear Boltzmann plots [46] and temperatures 30 to $50 \mathrm{~K}$ higher than expected [45, 47], respectively. Furthermore, it is generally known that in atmospheric-pressure helium plasmas, $\mathrm{He}_{2}{ }^{+}$is the dominant positive ion $[48,49]$ because of the efficient and fast ion conversion of $\mathrm{He}^{+}$into $\mathrm{He}_{2}^{+}$ through a three-body process [50]. Accordingly, we believe that the elevated $\mathrm{N}_{2}^{+}$temperatures $(\sim 850 \mathrm{~K})$ near the plate electrode, but before the maximum $\mathrm{N}_{2}{ }^{+}$emission, are due to charge-transfer ionization of atmospheric nitrogen with $\mathrm{He}_{2}^{+}$. As was suggested above, the maximum $\mathrm{N}_{2}^{+}$emission probably represents the point at which atmospheric nitrogen is no longer the limiting reagent. This behavior is seen also in the rotational temperature, when it reaches a constant value of $\sim 660 \mathrm{~K}$, as the point where much of the $\mathrm{He}_{2}{ }^{+}$is depleted.

\section{Conclusions}

The afterglow and APGD from the He-based FAPA desorption/ionization source were characterized through optical spectroscopy under a variety operating conditions to better understand factors that affect desorption and ionization for mass spectrometric analyses. The background emission spectrum of the APGD contained high-energy atomic helium lines, as well as molecular and atomic emission from contaminant species. Helium excimer, $\mathrm{He}_{2}{ }^{*}$, emission was also observed, which was not previously reported in spectroscopic studies of similar He-APGDs. Although not commonly mentioned in the ambient mass spectrometry literature, $\mathrm{He}_{2}{ }^{*}$ and other similar species, such as $\mathrm{He}_{2}{ }^{+}$, could contribute significantly to analyte ionization or reagent-ion formation. The spectrum of the afterglow in the open atmosphere did not contain emission features from any helium species, indicating that the majority of energetic helium moieties have either decayed to a state that emits outside of the measured spectral window (e.g., He* with $n=2$ or $\mathrm{He}^{\mathrm{m}}$ ) or exist in a form that does not radiatively decay (e.g., $\mathrm{He}_{2}{ }^{+}$).

Spatially resolved emission profiles of species in the APGD were employed to understand the role of operating parameters (discharge current, He flow rate, and discharge polarity) on production of excited atoms and molecules as 
well as to deduce the origin of impurities within the discharge region. Reducing the operating current from 25 to $2 \mathrm{~mA}$ transitioned the discharge from a true glow to more of a corona discharge, in which much of the emission and the majority of plasma species were created at the pin electrode, regardless of polarity. It is suggested that a substantial amount of $\mathrm{H}_{2} \mathrm{O}$ vapor, $\mathrm{O}_{2}$, and $\mathrm{N}_{2}$ enters the discharge chamber through diffusion from the atmosphere, rather than originating from the gas supply.

The emission profiles also revealed that the polarity of the electrodes affects the formation of species that control the sensitivity of the FAPA in mass spectrometric analyses. Specifically, the pin-positive configuration consistently yielded less intense emission, and thus a lower number density, for all examined species throughout the discharge compared with the pin-negative polarity. The gas-kinetic temperature, determined from the $\mathrm{OH}$ rotational temperature, was higher with a pin-negative polarity. Also, for both polarities, $\mathrm{N}_{2}^{+}$emission exhibited a maximum at the anode that slowly decayed into the positive column of the discharge, despite the fact that the gas flow moved from the pin electrode to the plate. This behavior implies that different chemistries and excitation conditions exist at the cathode and the anode. We believe an abundance of lowenergy electrons is present at the anode, which promotes the ionization of $\mathrm{He}^{*}$ and the formation of $\mathrm{He}_{2}{ }^{+}$, leading to the ionization of atmospheric constituents in an environment less energetic or harsh than in the negative glow at the cathode. Collectively, the findings explain why FAPA exhibits better sensitivity in a pin-negative configuration.

Lastly, spatial emission profiles of the afterglow of the FAPA revealed an emission maximum approximately $0.67 \mathrm{~mm}$ from the plate electrode. While the intensity of these emission features changed with discharge current and gas flow rate, the spatial location of the emission maximum was unaffected. Through thermographic data and $\mathrm{OH}$ rotational temperature values, the gas temperature of the afterglow under the present experimental conditions was found to be $\sim 500 \mathrm{~K}$. Interestingly, the $\mathrm{N}_{2}{ }^{+}$ rotational temperature reached a maximum of $860 \mathrm{~K}$ prior to the emission maximum. We believe this elevated $\mathrm{N}_{2}{ }^{+}$temperature is caused by charge-transfer ionization of $\mathrm{N}_{2}$ by $\mathrm{He}_{2}{ }^{+}$, as has also been shown with a helium dielectric-barrier discharge.

\section{Acknowledgments}

The authors acknowledge support in part for this research by the US Department of Energy through grant DE-FG0298ER14890 and by the National Science Foundation through grant CHE-0822114. The authors thank Horiba Jobin-Yvon for the loan of the ACTIVA Spectrometer used in this study.

\section{References}

1. Takats, Z., Wiseman, J.M., Gologan, B., Cooks, R.G.: Mass spectrometry sampling under ambient conditions with desorption electrospray ionization. Science 306, 471-473 (2004)
2. Venter, A., Nefliu, M., Cooks, R.G.: Ambient desorption ionization mass spectrometry. Trac-Trends Anal. Chem. 27, 284-290 (2008)

3. Van Berkel, G.J., Pasilis, S.P., Ovchinnikova, O.: Established and emerging atmospheric pressure surface sampling/ionization techniques for mass spectrometry. J. Mass Spectrom. 43, 1161-1180 (2008)

4. Weston, D.J.: Ambient ionization mass spectrometry: Current understanding of mechanistic theory; analytical performance and application areas. Analyst 135, 661-668 (2010)

5. Andrade, F.J., Shelley, J.T., Wetzel, W.C., Webb, M.R., Gamez, G., Ray, S.J., Hieftje, G.M.: Atmospheric pressure chemical ionization source. 1. Ionization of compounds in the gas phase. Anal. Chem. 80, 2646-2653 (2008)

6. Cody, R.B., Laramee, J.A., Durst, H.D.: Versatile new ion source for the analysis of materials in open air under ambient conditions. Anal. Chem. 77, 2297-2302 (2005)

7. Chen, H.W., Zheng, J., Zhang, X., Luo, M.B., Wang, Z.C., Qiao, X.L.: Surface desorption atmospheric pressure chemical ionization mass spectrometry for direct ambient sample analysis without toxic chemical contamination. J. Mass Spectrom. 42, 1045-1056 (2007)

8. Harper, J.D., Charipar, N.A., Mulligan, C.C., Zhang, X.R., Cooks, R. G., Ouyang, Z.: Low-temperature plasma probe for ambient desorption ionization. Anal. Chem. 80, 9097-9104 (2008)

9. Na, N., Zhao, M.X., Zhang, S.C., Yang, C.D., Zhang, X.R.: Development of a dielectric barrier discharge ion source for ambient mass spectrometry. J. Am. Soc. Mass Spectrom. 18, 1859-1862 (2007)

10. Ratcliffe, L.V., Rutten, F.J.M., Barrett, D.A., Whitmore, T., Seymour, D., Greenwood, C., Aranda-Gonzalvo, Y., Robinson, S., McCoustra, M.: Surface analysis under ambient conditions using plasma-assisted desorption/ionization mass spectrometry. Anal. Chem. 79, 6094-6101 (2007)

11. Symonds, J.M., Galhena, A.S., Fernandez, F.M., Orlando, T.M.: Microplasma discharge ionization source for ambient mass spectrometry. Anal. Chem. 82, 621-627 (2010)

12. Song, L., Gibson, S.C., Bhandari, D., Cook, K.D., Bartmess, J.E.: Ionization mechanism of positive-ion direct analysis in real time: A transient microenvironment concept. Anal. Chem. 81, 10080-10088 (2009)

13. Shelley, J.T., Hieftje, G.M.: Ionization matrix effects in plasma-based ambient mass spectrometry sources. J. Anal. At. Spectrom. 25, 345-350 (2010)

14. McEwen, C.N., Larsen, B.S.: Ionization mechanisms related to negative ion APPI, APCI, and DART. J. Am. Soc. Mass Spectrom. 20, 1518-1521 (2009)

15. Song, L.G., Dykstra, A.B., Yao, H.F., Bartmess, J.E.: Ionization mechanism of negative ion-direct analysis in real time: A comparative study with negative ion-atmospheric pressure photoionization. J. Am. Soc. Mass Spectrom. 20, 42-50 (2009)

16. Olenici-Craciunescu, S.B., Michels, A., Meyer, C., Heming, R., Tombrink, S., Vautz, W., Franzke, J.: Characterization of a capillary dielectric barrier plasma jet for use as a soft ionization source by optical emission and ion mobility spectrometry. Spectrochim. Acta Pt. B-Atom. Spectr. 64, 1253-1258 (2009)

17. Shelley, J.T., Wiley, J.S., Chan, G.C.Y., Schilling, G.D., Ray, S.J., Hieftje, G.M.: Characterization of direct-current atmospheric-pressure discharges useful for ambient desorption/ionization mass spectrometry. J. Am. Soc. Mass Spectrom. 20, 837-844 (2009)

18. Chan, G.C.Y., Shelley, J.T., Jackson, A.U., Wiley, J.S., Engelhard, C., Cooks, R.G., Hieftje, G.M.: Spectroscopic plasma diagnostics on a lowtemperature plasma probe for ambient mass spectrometry. J. Anal. At. Spectrom., 26, 1434-1444 (2011)

19. Chan, G.C.Y., Shelley, J.T., Wiley, J.S., Jackson, A.U., Engelhard, C., Cooks, R.G., Hieftje, G.M.: Elucidation of reaction mechanisms responsible for afterglow and reagent-ion formation in the low-temperature plasma probe ambient ionization source. Anal. Chem., 83, 3675-3686 (2011)

20. Chan, G.C.Y., Hieftje, G.M.: Warning indicators for the presence of plasma-related matrix effects in inductively coupled plasma-atomic emission spectrometry. J. Anal. At. Spectrom. 23, 181-192 (2008)

21. Andrade, F.J., Wetzel, W.C., Chan, G.C.Y., Webb, M.R., Gamez, G., Ray, S.J., Hieftje, G.M.: A new, versatile, direct-current helium atmospheric-pressure glow discharge. J. Anal. At. Spectrom. 21, 1175-1184 (2006)

22. Gielniak, B., Fiedler, T., Broekaert, J.A.C.: Study of a new direct current atmospheric pressure glow discharge in helium. Spectrochim Acta Pt. B-Atom. Spectr. 66, 21-27 (2011) 
23. Golubovskii, Y.B., Maiorov, V.A., Behnke, J., Behnke, J.F.: Modelling of the homogeneous barrier discharge in helium at atmospheric pressure. J. Phys. D-Appl. Phys. 36, 39-49 (2003)

24. Tsuji, M., Oda, E., Tanaka, M., Nakamura, M., Nishimura, Y.: Formation of the $\mathrm{He}_{2}(\mathrm{c}, \mathrm{d}, \mathrm{e}, \mathrm{f}, \mathrm{C}, \mathrm{D}, \mathrm{E}, \mathrm{F})$ states by ion-ion neutralization reaction of $\mathrm{He}_{2}{ }^{+}$with $\mathrm{C}_{6} \mathrm{~F}^{-}$in the helium flowing afterglow. Chem. Lett. 26, 465-466 (1997)

25. NIST Chemistry WebBook, http://webbook.nist.gov/chemistry/ (accessed December 6, 2010)

26. Martens, T., Mihailova, D., van Dijk, J., Bogaerts, A.: Theoretical characterization of an atmospheric pressure glow discharge used for analytical spectrometry. Anal. Chem. 81, 9096-9108 (2009)

27. Andrade, F.J., Shelley, J.T., Wetzel, W.C., Webb, M.R., Gamez, G., Ray, S.J., Hieftje, G.M.: Atmospheric pressure chemical ionization source. 2. Desorption-ionization for the direct analysis of solid compounds. Anal. Chem. 80, 2654-2663 (2008)

28. Laramee, J.A., Cody, R.B. Patent U.S. Pat. 7,112,785 B22006

29. Ascenzi, D., Franceschi, P., Guella, G., Tosi, P.: Phenol production in benzene/air plasmas at atmospheric pressure. Role of radical and ionic routes. J. Phys. Chem. A 110, 7841-7847 (2006)

30. Tosi, P., Ascenzi, D., Franceschi, P., Guella, G.: Ion chemistry in gaseous discharges at atmospheric pressure. Plasma Sources Sci. Tech. 18, 034005 (2009)

31. Nilles, J.M., Connell, T.R., Durst, H.D.: Thermal separation to facilitate direct analysis in real time (dart) of mixtures. Analyst 135, 883-886 (2010)

32. Dieke, G.H., Crosswhite, H.M.: The ultraviolet bands of $\mathrm{OH}-$ fundamental data. J. Quant. Spectrosc. Radiat. Transfer 2, 97-199 (1962)

33. Chidsey, I.L., Crosley, D.R.: Calculated rotational transition-probabilities for the A-X system of OH. J. Quant. Spectrosc. Radiat. Transfer 23, 187199 (1980)

34. Mermet, J.M.: In: Boumans, P.W.J.M. (ed.) Inductively Coupled Plasma Emission Spectroscopy-Part 2, pp. 353-386. Wiley-Interscience, New York (1987)

35. Kelleher, D.E., Wiese, W.L., Helbig, V., Greene, R.L., Oza, D.H.: Advances in plasma broadening of atomic-hydrogen. Phys. Scr. T 47, 75-79 (1993)

36. Torres, J., van de Sande, M.J., van der Mullen, J., Gamero, A., Sola, A.: Stark broadening for simultaneous diagnostics of the electron density and temperature in atmospheric microwave discharges. Spectrochim. Acta Pt. B-Atom. Spectr. 61, 58-68 (2006)
37. Lindholm, E.: Pressure broadening of spectral lines. Ark. Mat. Astron. Fys. 32A, 17 (1946)

38. Foley, H.M.: The pressure broadening of spectral lines. Phys. Rev. 69 616-628 (1946)

39. Gigosos, M.A., Gonzalez, M.A., Cardenoso, V.: Computer simulated Balmer- $\alpha$, $-\beta$, and $-\gamma$ Stark line profiles for non-equilibrium plasmas diagnostics. Spectrochim. Acta Pt. B-Atom. Spectr. 58, 1489-1504 (2003)

40. Kuraica, M.M., Konjevic, N.: Electric field measurement in the cathode fall region of a glow discharge in helium. Appl. Phys. Lett. 70, 15211523 (1997)

41. Videnovic, I.R., Konjevic, N., Kuraica, M.M.: Spectroscopic investigations of a cathode fall region of the grimm-type glow discharge. Spectrochim. Acta Pt. B-Atom. Spectr. 51, 1707-1731 (1996)

42. Bogaerts, A., Gijbels, R.: Comprehensive description of a Grimm-type glow discharge source used for optical emission spectrometry: A mathematical simulation. Spectrochim. Acta Pt. B-Atom. Spectr. 53, 437-462 (1998)

43. Bogaerts, A., Gijbels, R.: Two-dimensional model of a direct current glow discharge: Description of the argon metastable atoms, spattered atoms, and ions. Anal. Chem. 68, 2676-2685 (1996)

44. Shelley, J.T., Wiley, J.S., Hieftje, G.M.: Ultrasensitive ambient mass spectrometric analysis with a pin-to-capillary flowing atmosphericpressure afterglow source. Anal. Chem., 83, 5741-5748 (2011)

45. Endoh, M., Tsuji, M., Nishimura, Y.: Thermal-energy charge-transfer reactions $-\mathrm{He}_{2}^{+}$with $\mathrm{N}_{2}$ and CO. J. Chem. Phys. 79, 5368-5375 (1983)

46. Belikov, A.E.: Rotational and vibrational excitation of the $\mathrm{N}_{2}{ }^{+}$(B) state in a $\mathrm{He}+\mathrm{N}_{2}$ electron-beam plasma. Chem. Phys. 215, 97-109 (1997)

47. Richardson, W.C., Setser, D.W.: Penning ionization optical spectroscopy- metastable helium $\mathrm{He} 2{ }^{3} \mathrm{~S}$ atoms with nitrogen, carbon-monoxide, oxygen, hydrogen-chloride, hydrogen bromide, and chlorine. J. Chem. Phys. 58, 1809-1825 (1973)

48. Deloche, R., Monchicourt, P., Cheret, M., Lambert, F.: High-pressure helium afterglow at room temperature. Phys. Rev. A 13, 1140-1176 (1976)

49. Gerber, R.A., Sauter, G.F., Oskam, H.J.: Studies of decaying helium plasmas. Physica 32, 2173-2191 (1966)

50. Stevefelt, J., Pouvesle, J.M., Bouchoule, A.: Reaction-kinetics of a high-pressure helium fast discharge afterglow. J. Chem. Phys. 76, 4006-4015 (1982) 